Supporting information

\title{
Single-Layered $\mathrm{MoS}_{2}$ Directly Grown on Rutile $\mathrm{TiO}_{2}(110)$ for Enhanced Interfacial Charge Transfer
}

\author{
Huihui Liu, ${ }^{1, \dagger}$ Yue Li, ${ }^{2, \dagger}$ Miaomiao Xiang, ${ }^{1}$ Hualing Zeng, ${ }^{2,3,4, *}$ and Xiang Shao ${ }^{1,4,5, *}$ \\ ${ }^{1}$ Department of Chemical Physics, ${ }^{2}$ International Center for Quantum Design of Functional Materials \\ (ICQD), Hefei National Laboratory for Physical Sciences at the Microscale, ${ }^{3}$ Key Laboratory of \\ Strongly-Coupled Quantum Matter Physics, Chinese Academy of Sciences, Department of Physics, \\ ${ }^{4}$ Synergetic Innovation Center of Quantum Information and Quantum Physics, ${ }^{5}$ CAS Key Laboratory of \\ Urban Pollutant Conversion, University of Science and Technology of China, Hefei, Anhui 230026, China \\ ${ }^{\dagger}$ These two authors contribute equally to this work \\ *Towhom correspondence should be addressed: shaox@ustc.edu.cn, hlzeng@ustc.edu.cn
}




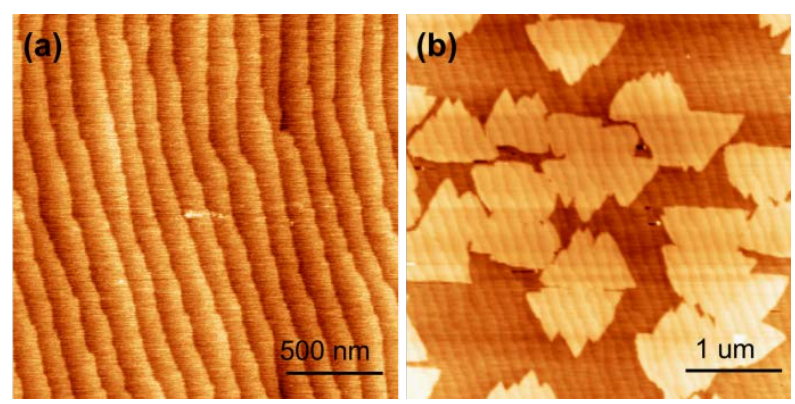

Figure S1. AFM topography images of (a) blank Nb-doped $\mathrm{TiO}_{2}(110)$ and (b) the ap- $\mathrm{MoS}_{2} / \mathrm{Nb}: \mathrm{TiO}_{2}$. Clearly the Nb doping has no influence on the structure of the fabricated $\mathrm{MoS}_{2}$.

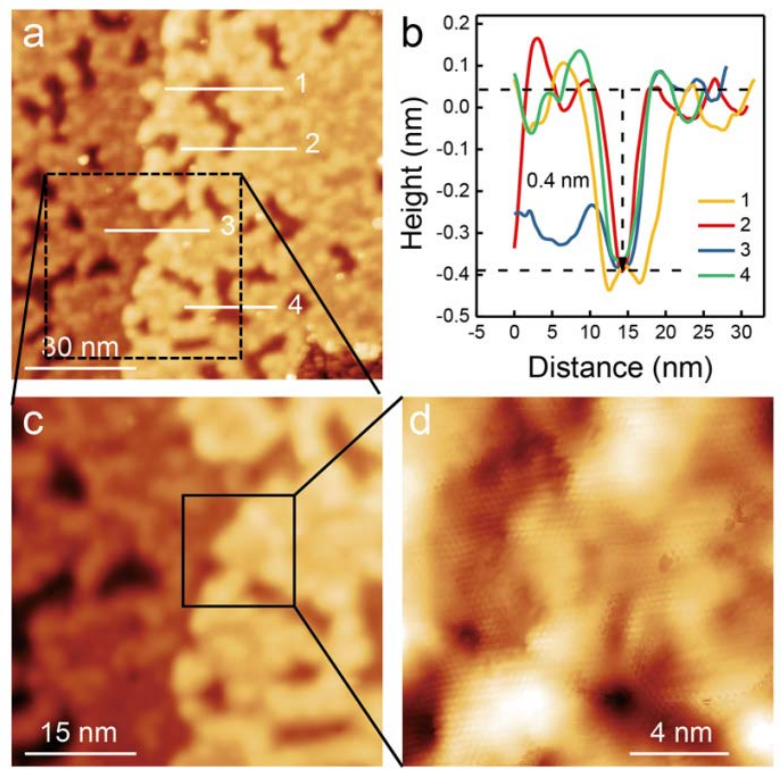

Figure S2. (a) STM image of the ap- $\mathrm{MoS}_{2} / \mathrm{TiO}_{2}(110)$ sample focusing on the step edge of $\mathrm{TiO}_{2}$. (b) Height profiles along the marked lines in (a), all showing the same corrugation as monostep height on $\mathrm{TiO}_{2}(110)$. (c) and (d) are STM images that are step-by-step magnification of the dashed box region in (a). The atomic resolution image shows continuous lattice of $\mathrm{MoS}_{2}$ when crossing the edges of the vacancies on $\mathrm{TiO}_{2}$ substrate. These images strongly support that the observed holes are the single-layer vacancies on $\mathrm{TiO}_{2}$ substrate. They also demonstrate that fabricated $\mathrm{MoS}_{2}$ attach closely with the $\mathrm{TiO}_{2}$ substrate. 


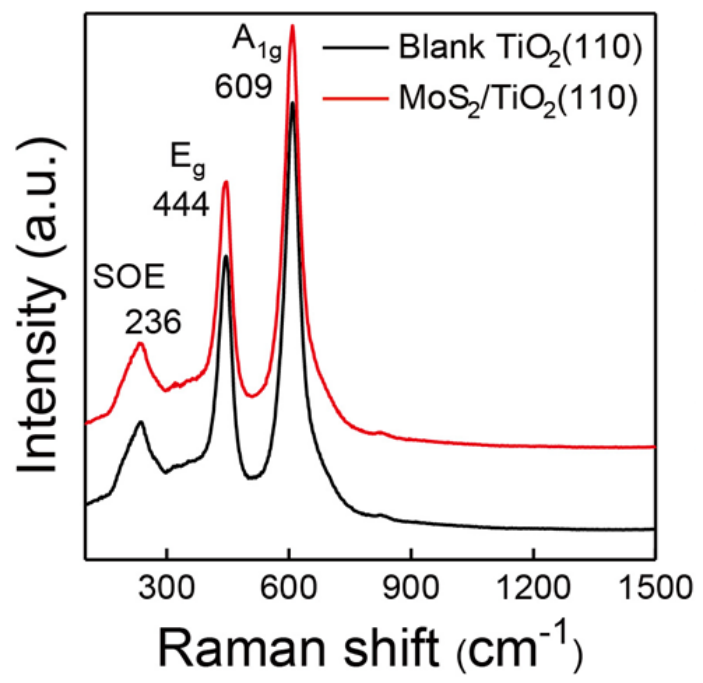

Figure S3. Comparison of Raman spectra of ap- $\mathrm{MoS}_{2} / \mathrm{TiO}_{2}$ and the blank $\mathrm{TiO}_{2}(110)$. The $\mathrm{MoS}_{2}$ signals are completely buried by the strong background of $\mathrm{TiO}_{2}$.

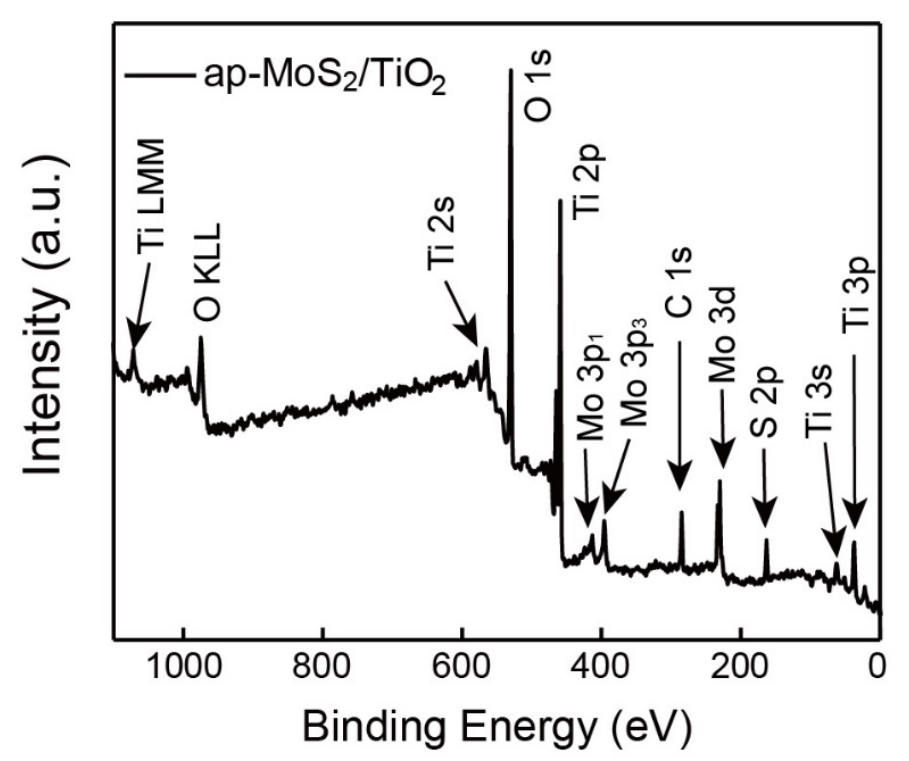

Figure S4. XPS survey spectrum of the ap- $\mathrm{MoS}_{2} / \mathrm{TiO}_{2}$ sample. 


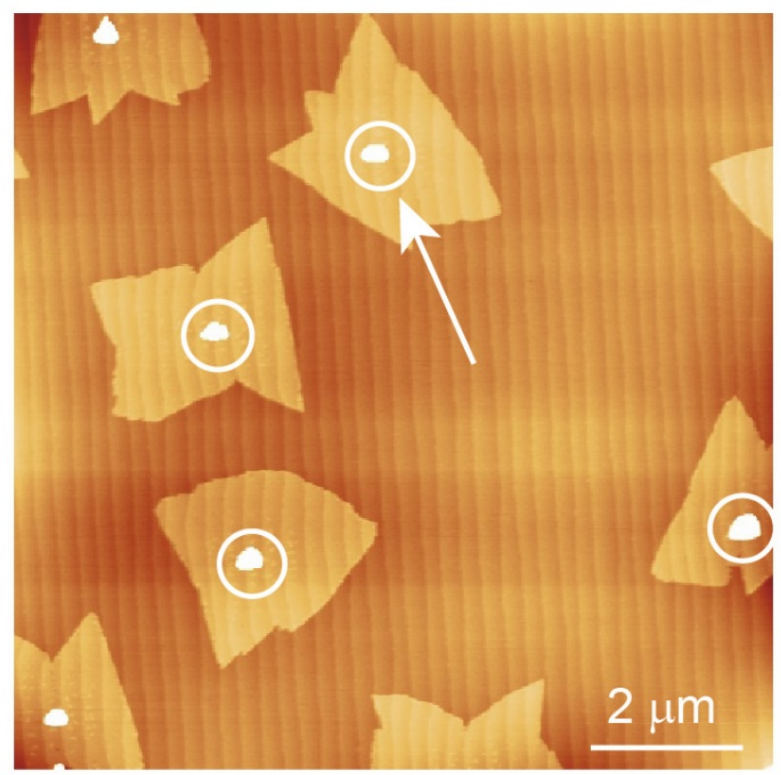

Figure S5. AFM image showing the unreacted $\mathrm{MoO}_{3}$ nucleus on ap- $\mathrm{MoS}_{2} / \mathrm{TiO}_{2}$ sample.

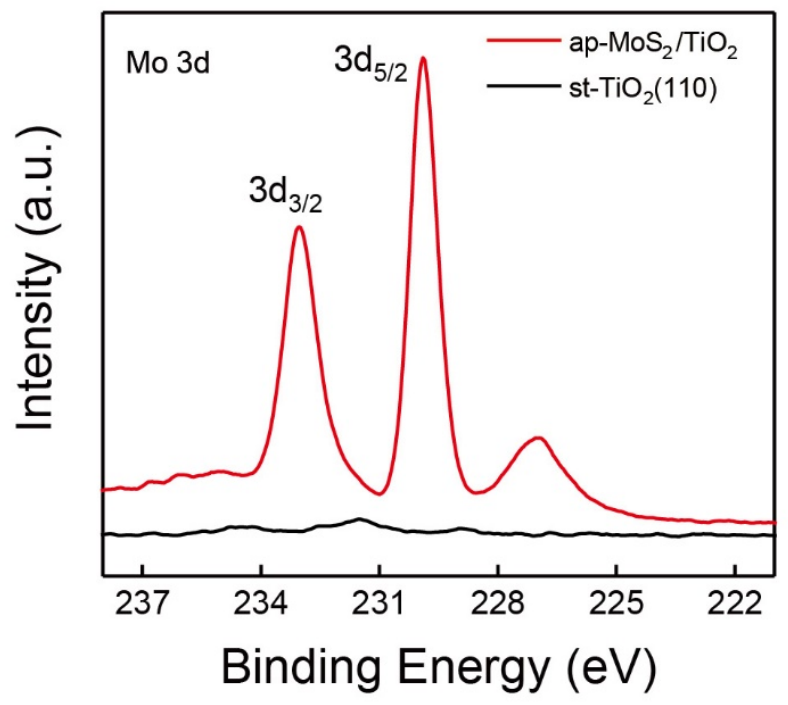

Figure S6. Comparison of Mo 3d XPS spectra of the ap- $\mathrm{MoS}_{2} / \mathrm{TiO}_{2}$ and st- $\mathrm{TiO}_{2}(110)$ samples. No residual Mo and S signals can be recognized after peeling off $\mathrm{MoS}_{2}$. 

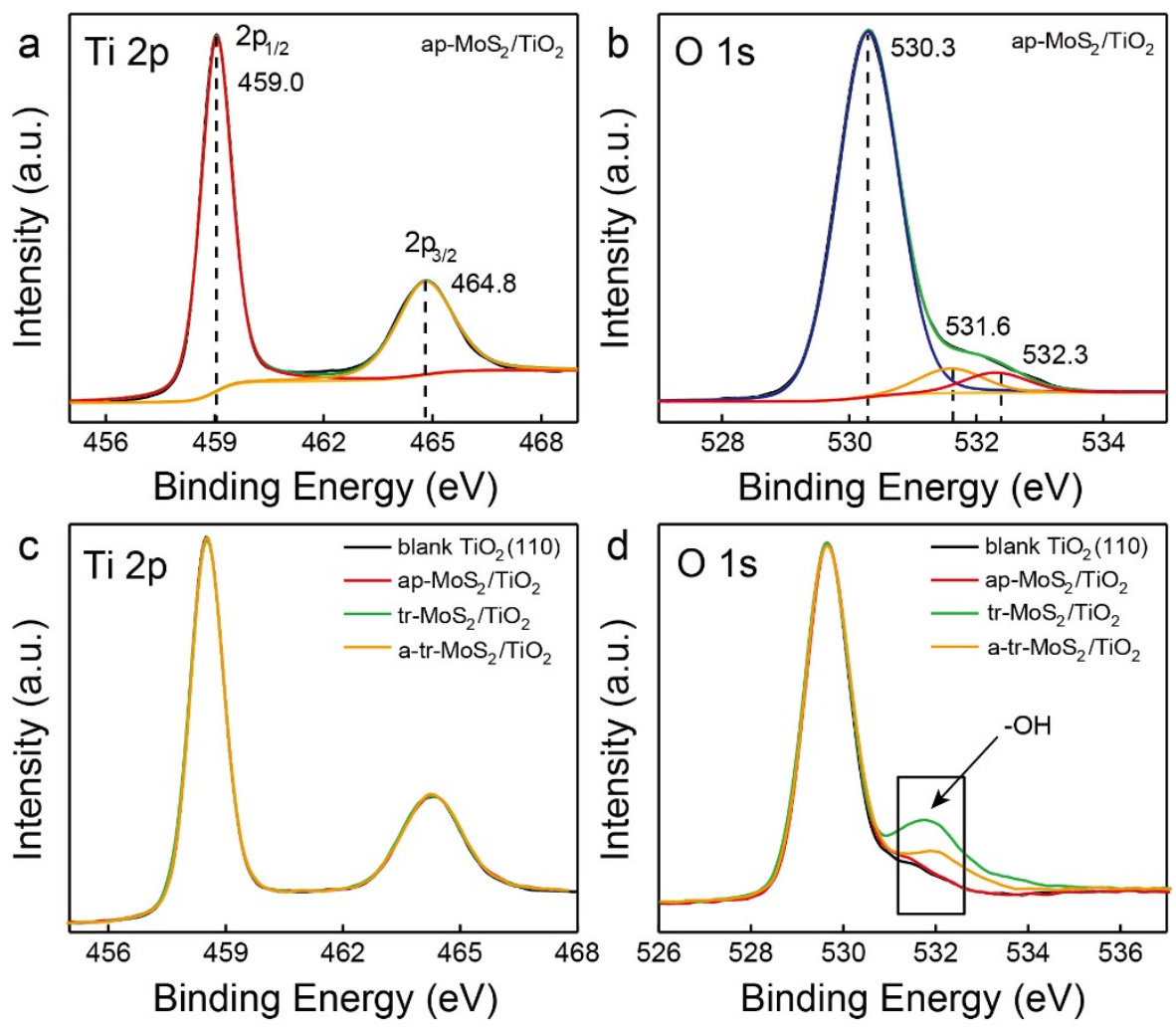

Figure S7. Deconvolution analyses of (a) Ti 2p and (b) O 1s spectra obtained on the ap- $\mathrm{MoS}_{2} / \mathrm{TiO}_{2}$ sample. No trace of $\mathrm{Ti} 3+$ species can be observed. The $\mathrm{O}$ 1s with B.E. of $531.6 \mathrm{eV}$ and $532.2 \mathrm{eV}$ can be assigned as $\mathrm{OH}$ and $\mathrm{CO}_{2}$ species adsorbed on the exposed $\mathrm{TiO}_{2}$ surface, respectively. (c) and (d) show the overlapping of the main peaks of $\mathrm{Ti} 2 \mathrm{p}$ and $\mathrm{O} 1 \mathrm{~s}$ of four different samples treated with simple normalization of the intensity combined with lateral shift. No detectable difference of Ti 2p can be found. Whereas for $\mathrm{O} 1 \mathrm{~s}$, significant amount of $\mathrm{OH}$ species was observed on the tr- $\mathrm{MoS}_{2} / \mathrm{TiO}_{2}$ sample. After annealing treatment, these species were largely yet incompletely removed. 

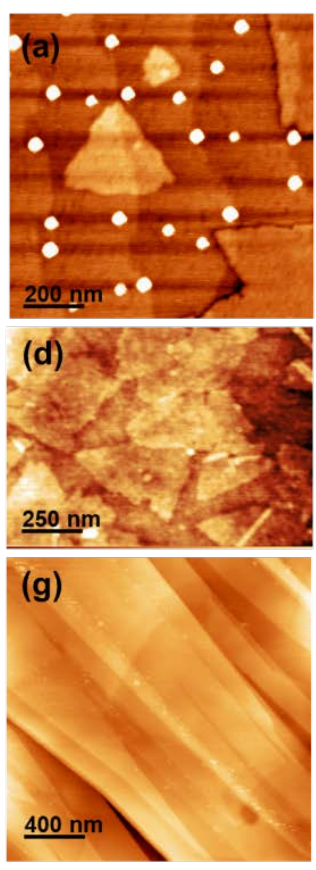
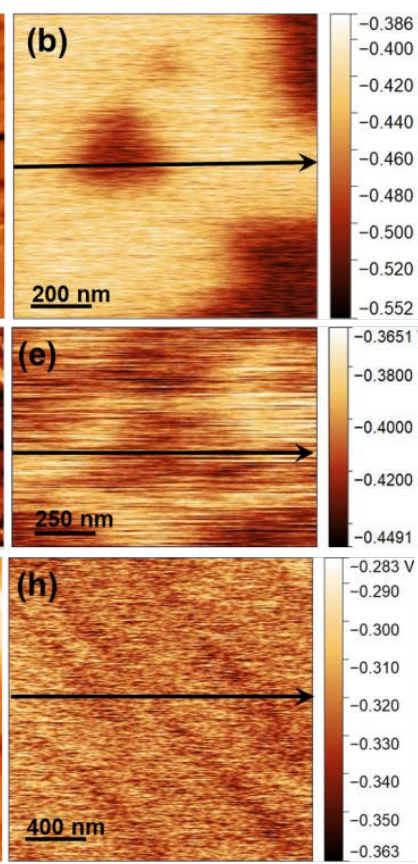
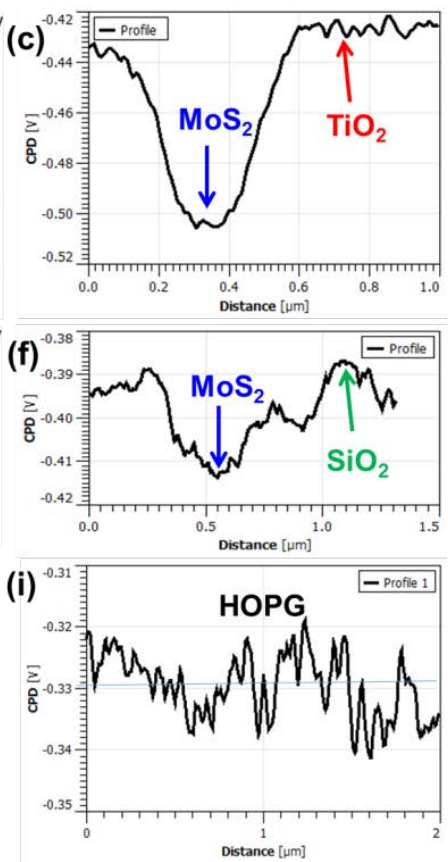

Figure S8. KPFM measurements on various sample surfaces with a distinct AFM tip against that used in Figure 5. (a-c) for $\mathrm{MoS}_{2} / \mathrm{TiO}_{2}$ sample, (d-f) for tr- $\mathrm{MoS}_{2} / \mathrm{SiO}_{2}$ sample, (g-i) for freshly cleaved HOPG. $(\mathrm{a}, \mathrm{d}, \mathrm{g})$ are topographic images, $(\mathrm{b}, \mathrm{e}, \mathrm{h})$ are corresponding surface potential images, $(\mathrm{c}, \mathrm{f}, \mathrm{i})$ are the surface potential profiles along the arrows.

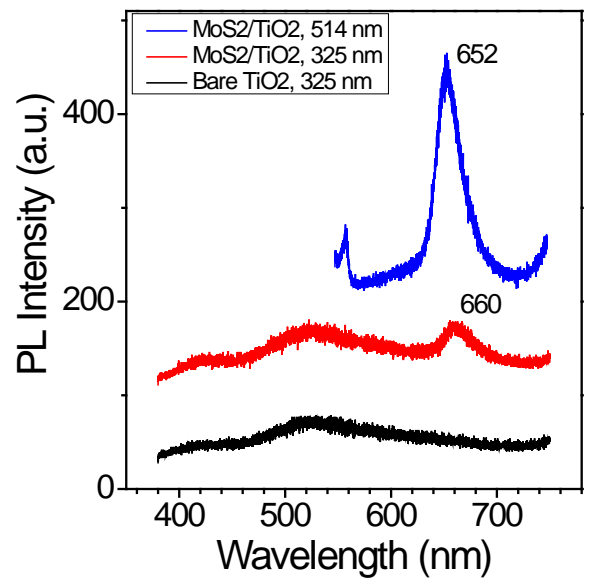

Figure S9. PL spectra taken on the $\mathrm{MoS}_{2}$ and the bare $\mathrm{TiO}_{2}$ regions, respectively, of an ap- $\mathrm{MoS}_{2} / \mathrm{TiO}_{2}$ sample under different excitations of incident lasers. Notice the power of the $325 \mathrm{~nm}$ laser is around $1 \mathrm{~mW}$ while that of the $514 \mathrm{~nm}$ laser is about $0.5 \mathrm{~mW}$. The PL peaks of $\mathrm{MoS}_{2}$ are marked. 

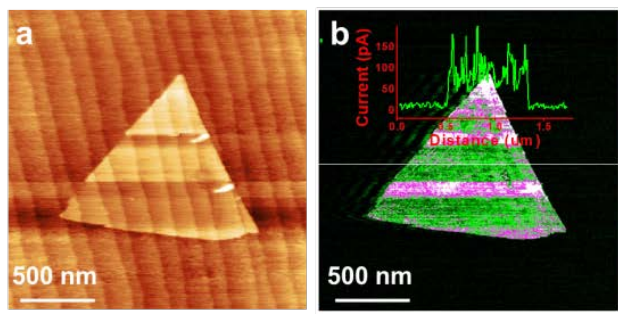

$\left[\begin{array}{r}250.0 \mathrm{pA} \\ 200.0 \\ 150.0 \\ 100.0 \\ 50.0 \\ 0.0\end{array}\right.$

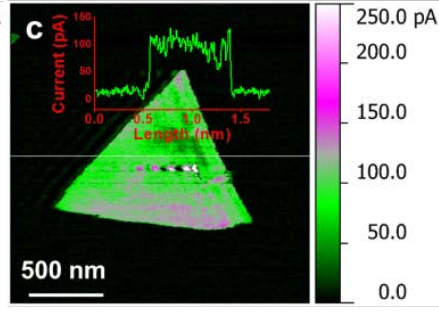

Figure S10. Topographic (a) and corresponding current images (b,c) of a selected $\mathrm{MoS}_{2}$ sheet obtained with Tunneling AFM mode in dark environment (b) and under laboratory illuminating light (c), respectively. The inserted profiles along the white line show the tunneling current in $\mathrm{MoS}_{2}$ remains around 100 pA disregarding the on/off state of the illumination torch whose spectrum is mostly in visible range, dictating a negligible conductivity change induced by $\mathrm{MoS}_{2}$ excitation. 